\title{
Cierre endoscópico de fístula gastrocutánea posterior al retiro de sonda de gastrostomía: reporte de caso
}

\author{
Raúl Antonio Zamarripa-Mottú, Enrique Murcio-Pérez*, Gerardo Blanco-Velasco, \\ Omar Michel Solórzano-Pineda y Óscar Víctor Hernández-Mondragón
}

Departamento de Endoscopia Gastrointestinal, Hospital de Especialidades Dr. Bernardo Sepúlveda Gutiérrez, Centro Médico Nacional Siglo XXI, Instituto Mexicano del Seguro Social, Ciudad de México, México

\section{Resumen}

En ocasiones, la indicación de la sonda de gastrostomía se resuelve y debe retirarse. Dentro de las complicaciones por retiro está la fístula gastrocutánea (FGC). Se presenta el caso de una paciente de 45 años con FGC posterior a retiro de sonda de gastrostomía, en quien se realizó cierre exitoso con dispositivo endoscópico Ovesco ${ }^{\circledR}$. Las terapias endoscópicas más efectivas son las que combinan desepitelización del trayecto fistuloso con método mecánico o monoterapia con Oves$\mathrm{CO}^{\oplus}$. Los dispositivos de cierre endoscópico tienen alta eficacia y seguridad como tratamiento de la FGC y deben considerarse como la primera opción de tratamiento.

Palabras clave: Fístula gastrocutánea. Cierre endoscópico. Ovesco ${ }^{\circledR}$.

\section{Endoscopic closure of gastrocutaneous fistula after gastrostomy tube removal: case report}

\begin{abstract}
Occasionally, the indication of the gastrostomy tube is resolved, and it is removed. Among the complications due to withdrawal is the gastro-cutaneous fistula (GCF). We present the case of a 45-year-old woman with GCF after gastrostomy catheter removal, in whom a successful closure with an Ovesco $0^{\circledR}$ endoscopic device was performed. The most effective endoscopic therapies are those that combine de-epithelization of the fistulous tract with mechanical method or monotherapy with Oves$\mathrm{co}^{\oplus}$. Endoscopic closure devices have high efficacy and safety as an FGC treatment and should be considered as the first treatment option.
\end{abstract}

Key words: Gastro-cutaneous fistula. Endoscopic closure. Ovesco ${ }^{\circledR}$.

\section{Correspondencia:}

*Enrique Murcio-Pérez

Av. Cuauhtemoc, 330

Col. Doctores, Del. Cuautémoc

Fecha de recepción: 01-02-2019

C.P. 06720, Ciudad de México, México

E-mail: murcio@ hotmail.com
Disponible en internet: 06-09-2019

Endoscopia. 2019;31:118-124

www.endoscopia-ameg.com 


\section{Introducción}

La colocación de sonda de gastrostomía es una forma segura y eficaz para nutrición enteral en pacientes que no toleran la alimentación vía oral pero tienen un tracto gastrointestinal intacto ${ }^{\dagger}$. Debido a su alta tasa de éxito y menor número de complicaciones en comparación con su colocación quirúrgica, se considera que la colocación endoscópica es el método de primera elección ${ }^{2}$.

En ocasiones, la indicación de la sonda de gastrostomía se resuelve y esta puede retirarse mediante tracción externa. Habitualmente el orificio cierra espontáneamente en 48 a $72 \mathrm{~h}^{3}$. Dentro de las complicaciones relacionadas con su retiro está la fístula gastrocutánea $(\mathrm{FGC})^{4}$, que se define como aquel trayecto entre la cámara gástrica y la piel que persiste después de cuatro semanas del retiro de la sonda de gastrostomía ${ }^{5}$. La incidencia de formación de FGC posterior a retiro de sonda de gastrostomía es baja $(0.29 \%)^{6}$. Cuando esto ocurre, existen diferentes alternativas endoscópicas para su cierre. Se presenta el caso de una paciente con FGC posterior a retiro de sonda de gastrostomía en quien se realizó cierre exitoso con un dispositivo endoscópico.

\section{Caso clínico}

Mujer de 45 años de edad que fue intervenida quirúrgicamente en 2016 por un tumor cerebral de $17 \mathrm{~cm}$, $\sin$ otros antecedentes relevantes. El reporte histopatológico fue compatible con meningioma grado I de la Organización Mundial de la Salud. Debido a la magnitud de la intervención, la paciente no tenía capacidad de deglución, por lo que fue colocada sonda de gastrostomía percutánea asistida por endoscopia en el mismo internamiento. La paciente realizó rehabilitación neurológica y logró recuperar, desde mediados de 2018, la capacidad de deglución y movilidad. Desde entonces la totalidad de su ingesta calórica era vía oral y manifestó su deseo de que le fuera retirada la sonda, misma que se retiró mediante tracción en septiembre de 2018. Un mes posterior a su retiro, la paciente refería salida continua de gas y líquido por orificio externo de FGC que condicionaba eritema y ardor en la piel circundante (Fig. 1).

Debido a lo anterior se propuso intento de cierre endoscópico con dispositivo over the scope clip (Oves$\mathrm{Co}^{\circledR}$, Tübingen, Alemania) tipo GC como se explica a continuación. En un primer tiempo se avanzó hasta la cámara gástrica endoscopio de visión frontal con canal

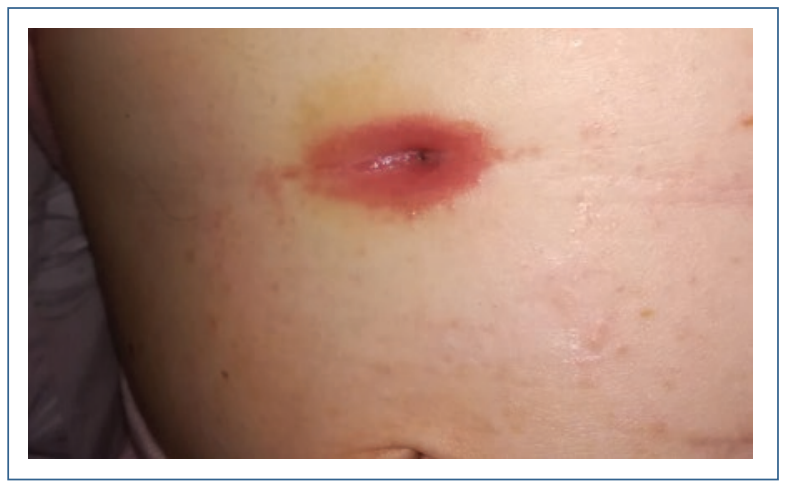

Figura 1. Aspecto externo de la fístula gastrocutánea previo a cierre endoscópico.

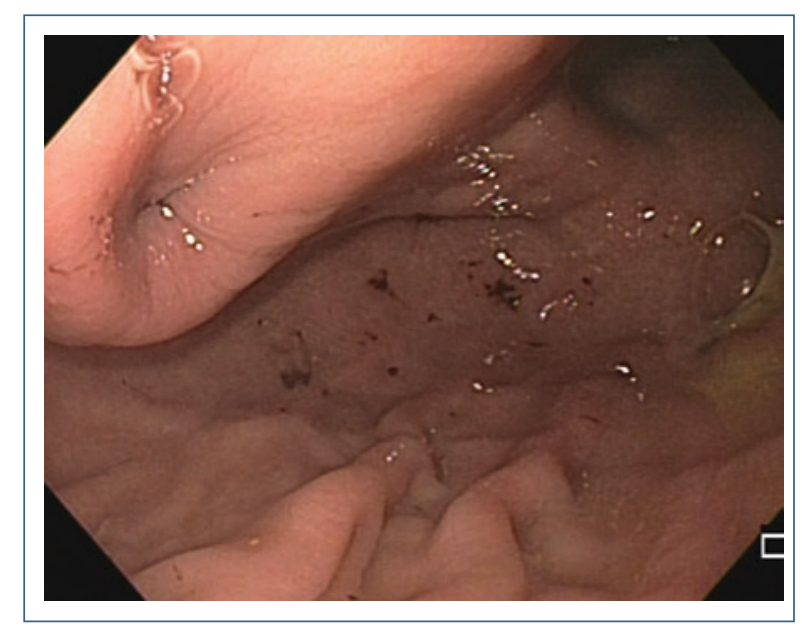

Figura 2. Aspecto interno de la fístula gastrocutánea previo a cierre endoscópico.

de trabajo de $2.8 \mathrm{~mm}$ (Olympus ${ }^{\circledR}$, GlF180). Se identificó orificio interno de fístula en cara anterior de cuerpo gástrico (Fig. 2). El trayecto fistuloso fue desepitelizado mediante ablación térmica utilizando sonda bipolar calibre $7 \mathrm{fr}$ (GoldProbe ${ }^{\circledR}$, Boston Scientific), con unidad electroquirúrgica Erbe $\mathrm{VIO}^{\circledR}$ 200s (Erbe Elektromedizin) modo soft coag con corriente de $20 \mathrm{w}$ con aplicaciones por ciclos de 6 a $8 \mathrm{~s}$ cada una hasta lograr coloración blanquecina uniforme del epitelio (Figs. 3 y 4). Tras la ablación térmica se introdujo en múltiples ocasiones cepillo de citología (Cytomax $\|^{\circledR}$ Double Lumen Cytology Brush, Wilson-Cook Medical, Ireland) por el trayecto fistuloso hasta observar discreto sangrado (Fig. 5). Se retiró el endoscopio y se colocó cierre de dispositivo Ovesco ${ }^{\circledR}$ tipo GC. En primera instancia, con auxilio de pinza ancla (OTSC ${ }^{\circledR}$ Anchor, Alemania) se 


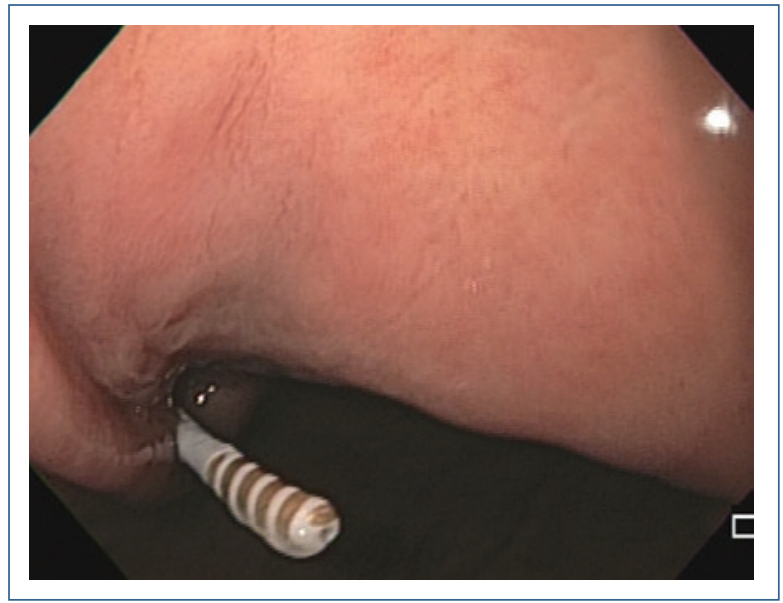

Figura 3. Sonda bipolar, pasa a través de orificio fistuloso desde el lado cutáneo, bajo visión endoscópica.

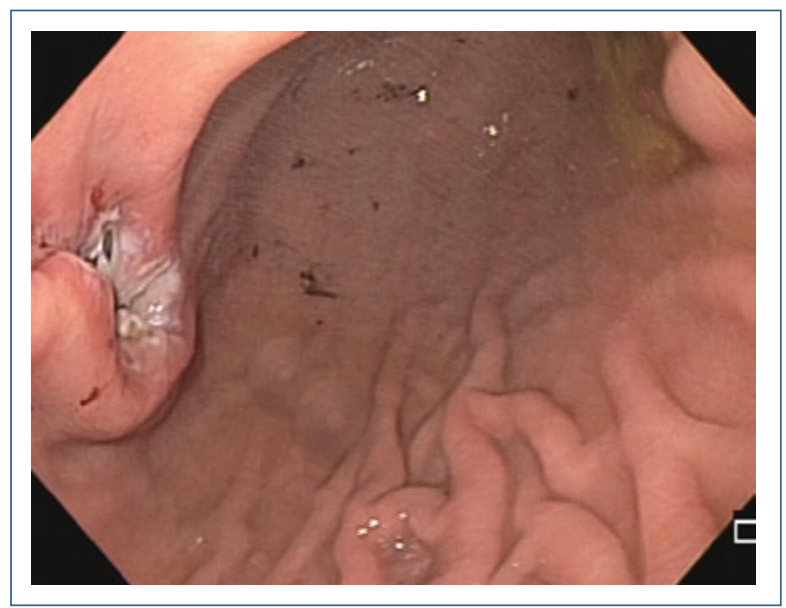

Figura 4. Aspecto de fístula gastrocutánea posterior a termoablación.

intentó la introducción del orificio interno dentro del dispositivo Ovesco ${ }^{\circledR}$. Sin embargo, la presencia de la pinza dentro del canal de trabajo del endoscopio disminuía notablemente el poder de aspiración e impedía un adecuado acoplamiento del orificio interno dentro del dispositivo Ovesco ${ }^{\circledR}$. Por lo anterior, se retiró pinza ancla y con auxilio de guía hidrofílica de $1 \mathrm{~mm}$ se centró dispositivo de cierre endoscópico, aplicándose de forma exitosa mediante aspiración (Figs. 6 y 7). La paciente tuvo cese inmediato de salida de gas y líquido por orificio externo de fístula. La revisión siete días después mostró resolución (Fig. 8). Tras seis meses de seguimiento, la paciente permanece sin evidencia de recurrencia de FGC.

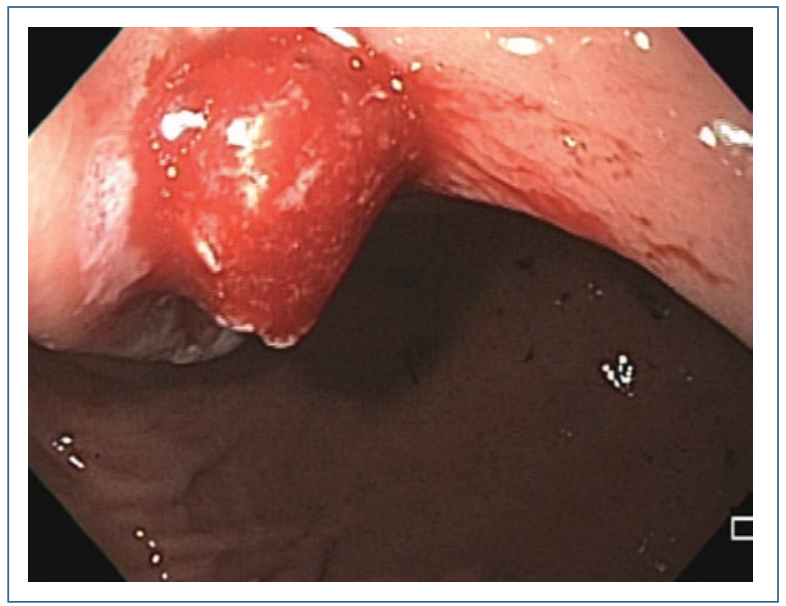

Figura 5. Lado gástrico de fístula posterior a desepitelización con cepillo de citología.

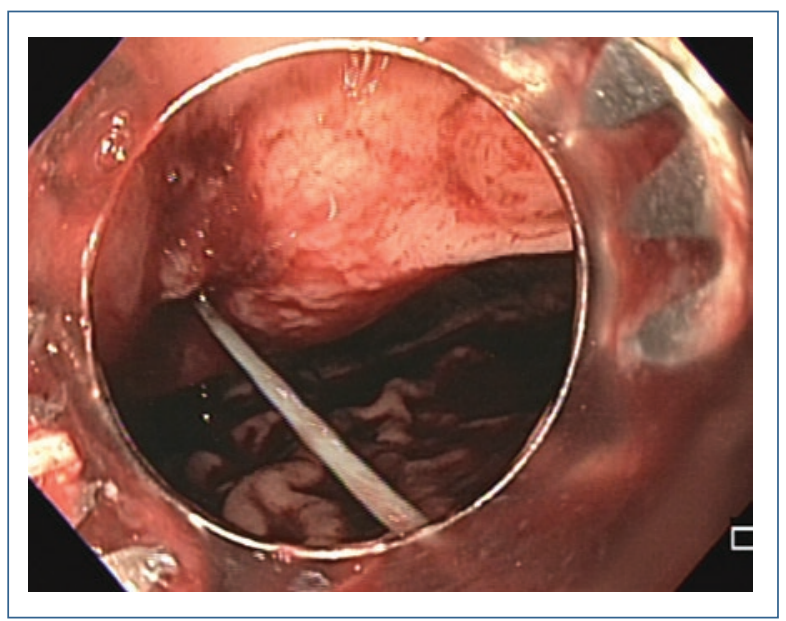

Figura 6. Guía hidrofílica a través de fístula para auxilio de aplicación de Ovesco ${ }^{\circledR}$.

\section{Discusión}

Las FGC posteriores a retiro de sonda de gastrostomía se deben sospechar en los pacientes que presentan salida de líquido, aire o celulitis en sitio de salida de sonda posterior a cuatro semanas del retiro de la sonda de gastrostomía. Los factores que predisponen al desarrollo de FGC refractarias son: pobre curación del tejido, retraso en el vaciamiento gástrico e incremento del flujo de contenido gástrico a través de la fístula y la fibrosis que acompaña invariablemente al tejido lesionado de la FGC ${ }^{5,7}$.

Se han analizado los factores de riesgo asociados a formación de FGC, y el tiempo entre la colocación y el 


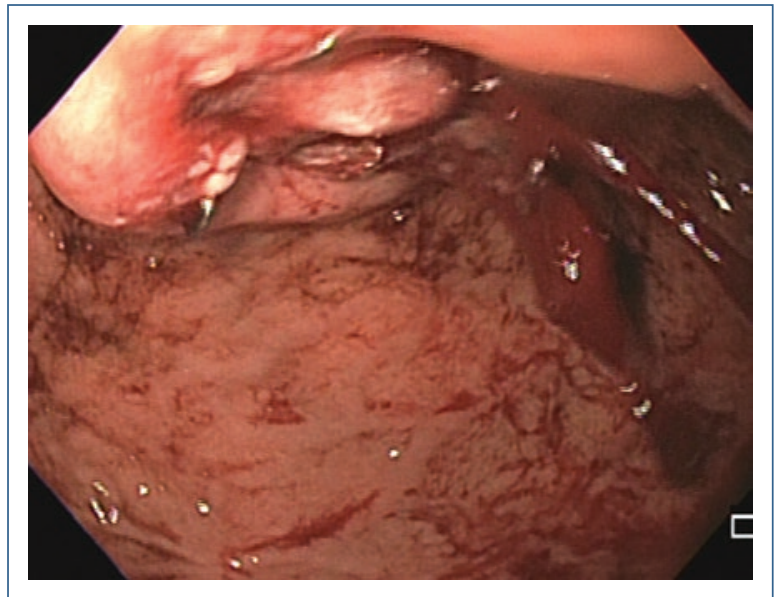

Figura 7. Clip Ovesco ${ }^{\circledR}$ colocado de forma exitosa, con cierre inmediato de fístula.

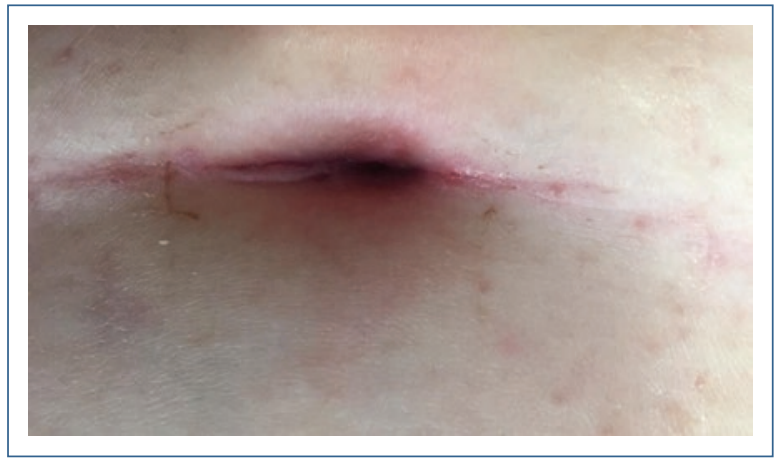

Figura 8. Aspecto cutáneo de fístula gastrocutánea, una semana posterior a cierre endoscópico.

retiro de la sonda de gastrostomía es el único factor de riesgo independiente para la incidencia de FGC. Lo anterior quedó demostrado en un estudio retrospectivo publicado en 2013 por Wyrick, et al., donde encontraron que los pacientes con gastrostomía durante 303 días tuvieron una incidencia calculada del $23 \%$, con aumento al $45 \%$ al permanecer 540 días $(p<0.001)$ y al $50 \%$ a los 850 días $(p<0.011)$. En el caso presentado la paciente tenía más de 850 días de la colocación de la sonda de gastrostomía.

Otro factor de riesgo considerado es el tipo de abordaje utilizado para su colocación: vía quirúrgica vs. vía endoscópica. Al respecto, un metaanálisis publicado en 2016 no encontró diferencia significativa en la incidencia de FGC si se coloca vía quirúrgica o vía endoscópica (razón de momios 1.07, intervalo de confianza 95\%: 0.485-2.351); sin embargo, previamente se encontró que, dentro del grupo de colocación quirúrgica, la incidencia aumenta si se coloca mediante cirugía abierta al compararlo con vía laparoscópica (incidencia: $43 \%$ en el grupo quirúrgico vs. $22 \%$ en el grupo laparoscópico, $p=0.004)^{6,8}$.

La reintervención quirúrgica en las complicaciones posquirúrgicas tiene alta morbimortalidad, prolonga la estancia hospitalaria e incrementa el costo ${ }^{9}$. En el caso de la FGC, la reparación mediante incisión abierta tiene una tasa alta de éxito, aunque puede cursar con infección local e infección de la herida quirúrgica, lo que predispone a los pacientes a recurrencia de la fístula ${ }^{10}$.

El uso de fármacos inhibidores del ácido gástrico no ha mostrado beneficio en favorecer un cierre espontáneo de la FCG. En un estudio retrospectivo pediátrico se incluyeron 97 niños en tratamiento con ranitidina, inhibidor de bomba de protones 0 ambos. No hubo diferencia en la frecuencia de cierre de la FGC (ranitidina $p=0.09$, inhibidor de bomba de protones $p=0.83$ y ambos $p=0.06$, respectivamente) ${ }^{11}$.

Con el desarrollo de la endoscopia intervencionista se han descrito distintos métodos endoscópicos para el cierre de la FGC en adultos con grados de eficacia variable, como se muestra en la tabla 1. Dentro de los múltiples casos reportados, destaca que las terapias más efectivas son las que combinan la desepitelización del trayecto fistuloso con un método endoscópico mecánico o la monoterapia con Ovesco ${ }^{\circledR}$ y que el cierre con clips o ligadura como monoterapia presenta alta tasa de recurrencia. Por lo anterior, entre las cuestiones técnicas a considerar antes del cierre de la FGC se recomienda la desepitelización del trayecto fistuloso. Para dicho fin se han utilizado diferentes dispositivos mecánicos (cepillos) y térmicos (argón plasma, sonda bipolar).

Se han descrito múltiples dispositivos endoscópicos para el cierre de FGC. Se han utilizado adhesivos tisulares (cianoacrilato, fibrina), clips convencionales, dispositivos de ligadura endoscópica, dispositivos Ovesco ${ }^{\circledR}$ y hasta métodos más sofisticados de sutura endoscópica. Sin embargo, al día de hoy no se cuenta con ensayos clínicos comparativos entre los diferentes métodos endoscópicos para el cierre de FGC. En México, la disponibilidad de los dispositivos previamente descritos es limitada, por lo que las condiciones de cada medio podrían ser determinantes en la elección de la herramienta endoscópica de cierre.

\section{Conclusión}

Los dispositivos de cierre endoscópico tienen alta eficacia y seguridad en el tratamiento de la FGC, por 


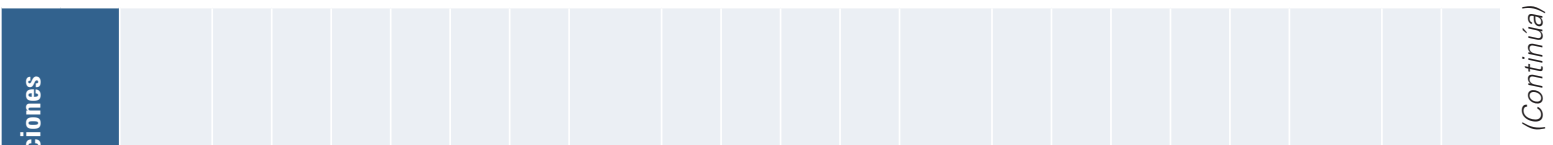

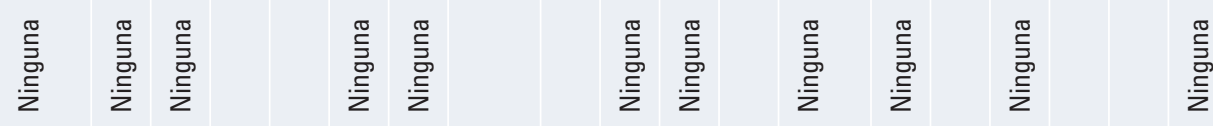
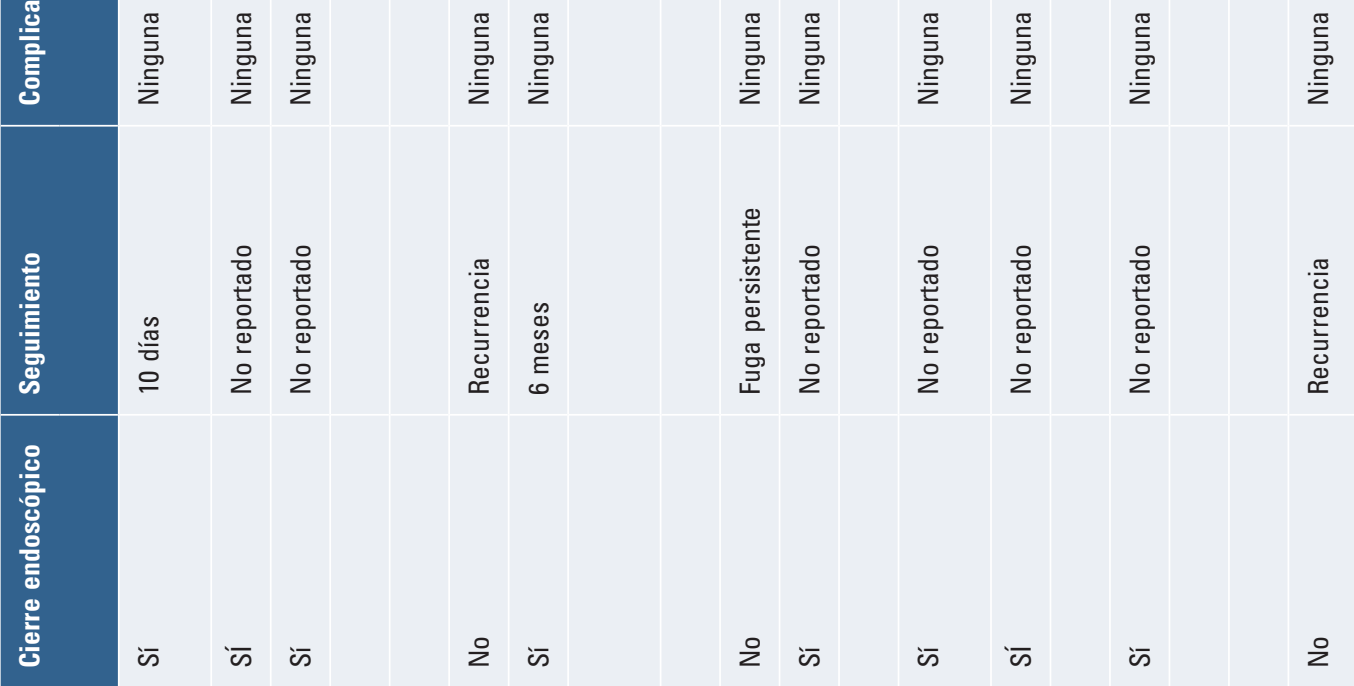

$\frac{2}{2}$ is 2 is is is is $\frac{2}{2}$

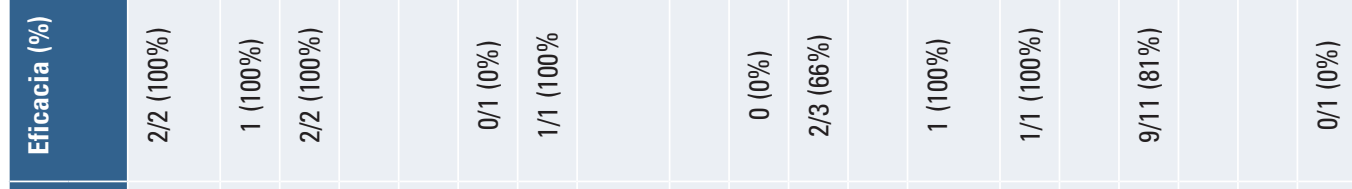

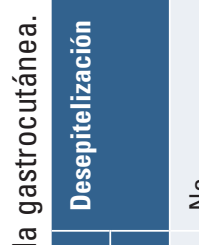

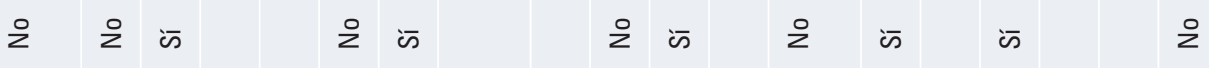

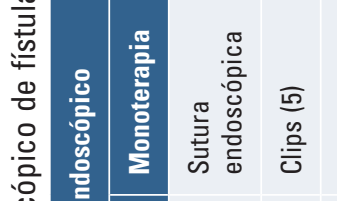

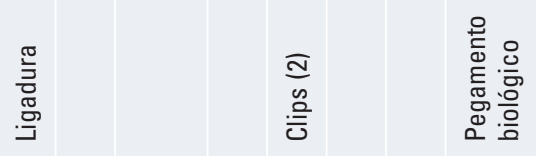

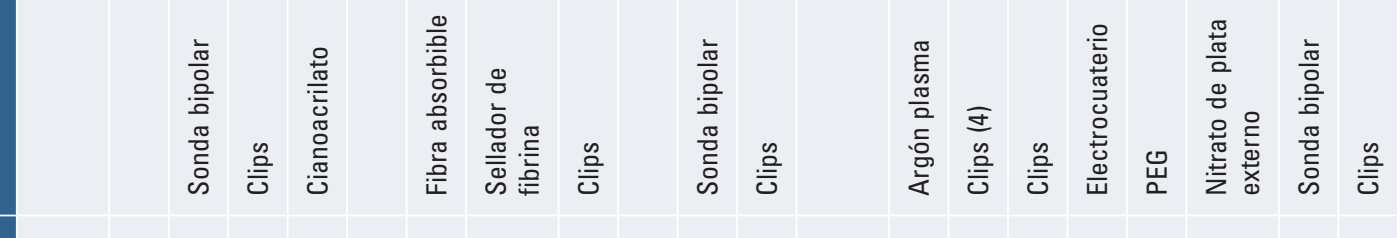

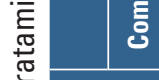

离

要

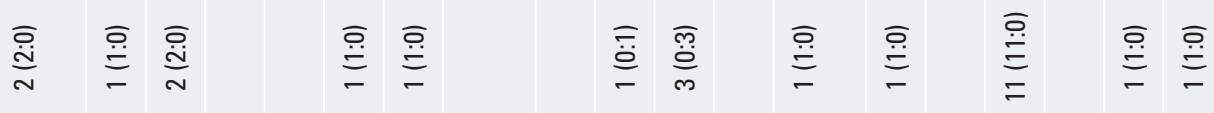

学

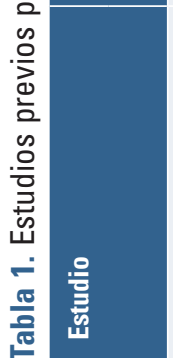

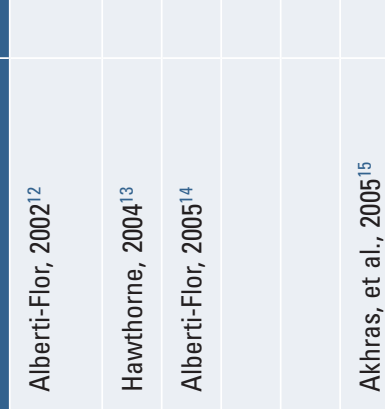

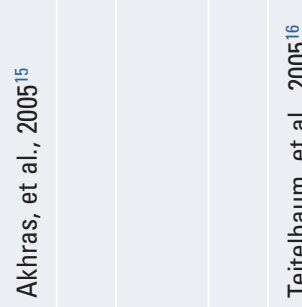

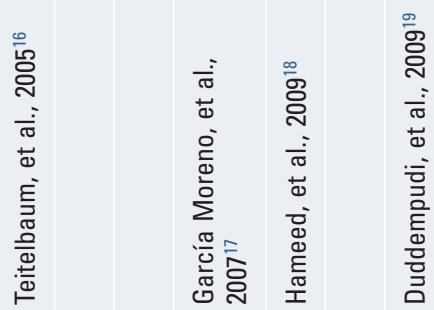

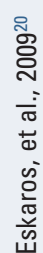




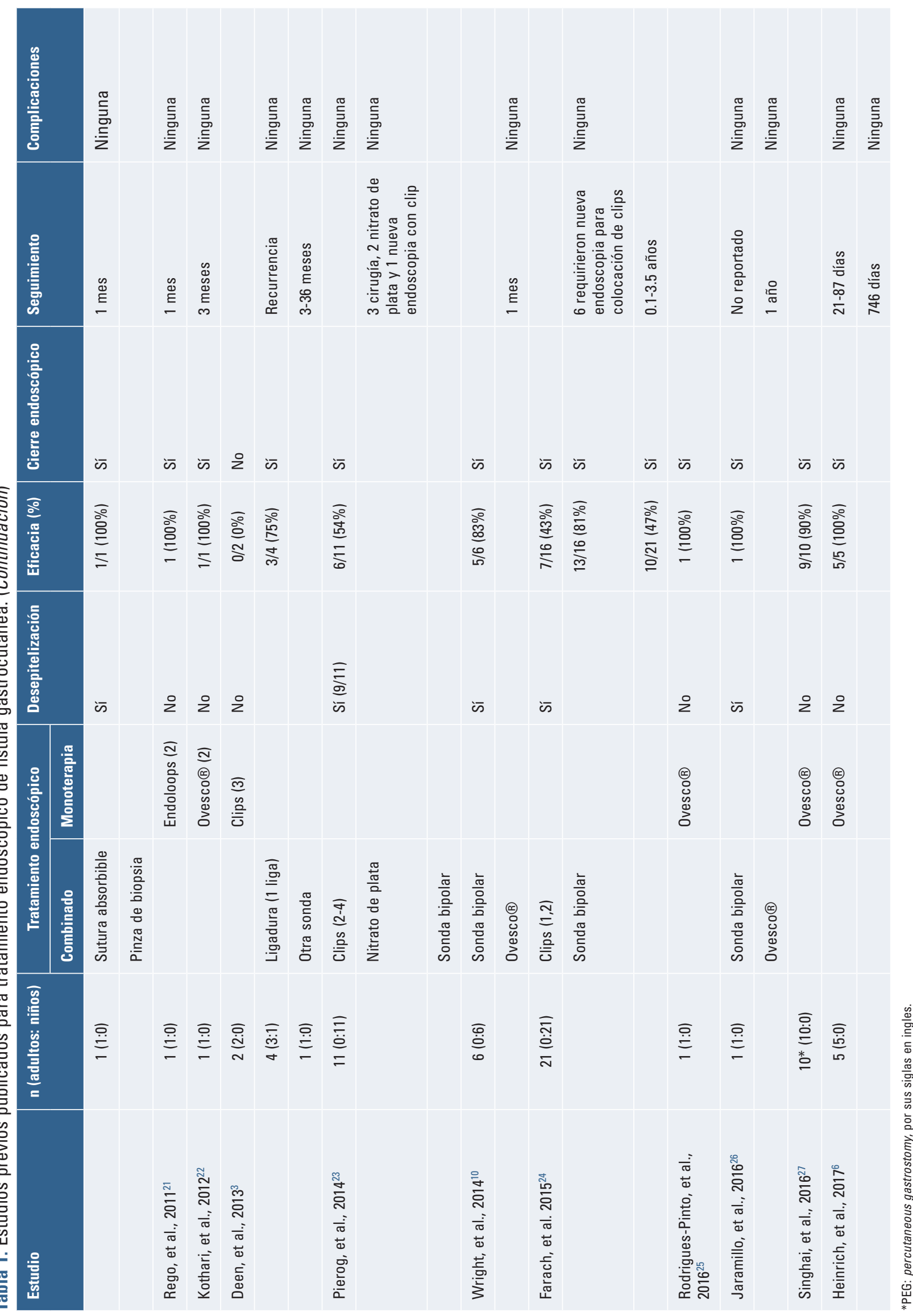




\section{lo que deben ser considerados como la primera opción de tratamiento.}

\section{Bibliografía}

1. Gauderer MW, Ponsky JL, Izant RJ Jr. Gastrostomy without laparotomy: a percutaneous endoscopic technique. J Pediatr Surg. 1980;15:872-875.

2. Duszak R Jr, Mabry MR. National trends in gastrointestinal access procedures: an analysis of Medicare services provided by radiologists and other specialists. J Vasc Interv Radiol. 2003;14:1031-6.

3. Deen OJ, Parisian KR, Harris C $3^{\text {rd }}$, Kirby DF. A novel procedure for gastrocutaneous fistula closure. J Clin Gastroenterol. 2013:47; 608-11.

4. Janik TA, Hendrickson RJ, Janik JS, Landholm AE. Analysis of factors affecting the spontaneous closure of a gastrocutaneous fistula. J Pediatr Surg. 2004;39:1197-9.

5. Heinrich H, Gluber C, Valli P. Over-the-scope-clip closure of long lasting gastrocutaneous fistula after percutaneous endoscopic gastrostomy tuve removal in immunocompromised patients: A single center case series. World J Gastrointest Endosc. 2017;9(2):85-90.

6. St-Louis E, Safa N, Guadagno E y Baird R. Gastrocutaneous fistulae in children - A systematic review and meta-analysis of epidemiology ans treatment options. J Pediatr Surg. 2018;53(5):946-58.

7. Gordon J, Langer J. Gastrocutaneous fistula in children after removal of gastrostomy tube: incidence and predictive factors. J Pediatr Surg 1999;34:1345-6.

8. Wyrick DL, Bozeman AP, Smith SD, Jackson RJ, Maxson RT, Kelley KR et al. Persistent gastrocutaneous fistula: Factors affecting the need for closure. J Pediatr Surg. 2013;48(12):2506-10.

9. Gordon J, Langer J. Gastrocutaneous fistula in children after removal of gastrostomy tube: incidence and predictive factors. J Pediatr Surg. 1999; 34:1345-6.

10. Wright R, Abrajano C, Koppolu R, Stevens M, Nyznyk S, Chao S, et al. Initial results of endoscopic gastrocutaneous fistula closure in children using an over-the-scope clip. J Laparoendosc Adv Surg Tech A. 2015; 25(1):69-72.

11. Thomas PG, Sharp NE, Schnell K, Snyder CL, St Peter SD. The role of medication in spontaneus gastrocutaneous fistula closure. Eur J Pediatr Surg. 2015;25:405-8.

12. Alberti-Flor JJ. Percutaneous-endoscopic suturing of gastrocutaneous fistula: report of 2 cases. Gastrointest Endosc. 2002:56(5):751-3.

13. Hawthorne AB. Endoscopic clipping of a nonhealing gastrocutaneous fistula following gastrostomy removal. Endoscopy. 2004;36:834
14. Alberti-Flor JJ. Endoclip-assisted closure of infected gastrocutaneous fistulas: Report of two cases. Endoscopy. 2005;37:403.

15. Akhras J, Tobi M, Zagnoon A. Endoscopic fibrin sealant injection with application of hemostatic clips: A novel method of closing a refractory gastrocutaneous fistula. Dig Dis Sci. 2005;50(10):1872-4

16. Teitelbaum JE, Gorcey SA, Fox VL. Combined endoscopic cautery and clip closure of chronic gastrocutaneous fistulas. Gastrointest Endosc. 2005;62(3):432-5

17. García Moreno J, Suárez Grau JM, Gómez Bravo M, Álamo Martínez J, Domínguez Usero D, Valera Sánchez Z, et al. Fístula gastrocutánea resuelta mediante inyección endoscópica de cola biológica. Rev Esp Enferm Dig. 2007;99(11):672-80.

18. Hameed $\mathrm{H}$, Kalim S, Khan Yi. Closure of a nonhealing gastrocutaneous fistula using argon plasma coagulation and endoscopic hemoclips. Can J Gastroenterol. 2009;23(3):217-9.

19. Duddempudi S, Ghevariya V, Singh M, Kishnaiah M. Treatment of persistenly leaking post PEG tube gastrocutaneous fistula in elderly patients with combined electrochemical cautery and endoscopic clip placement. South Med J. 2009;102(6):585-8.

20. Eskaros S, Ghevariya V, Krishnaiah M, Asarian A, Anand S. Percutaneous endoscopic suturing: an effective treatment for gastrocutaneous fistula. Gastrointest Endosc. 2009;70(4):768-71.

21. Rego AC, Nunes N, Pereira JR, Paz N, Duarte MA. Double endoloop technique for hole closure on the gastrointestinal tract: Human application. Clin Gastroenterol Hepatol. 2011;9(10):e104-5.

22. Kothari TH, Haber G, Sonpal N, Karanth N.The over-the scope clip system - a novel technique for gastrocutáneous fístula closure: The first North American experience. Can J Gastroenterol. 2012;26(4):193-5.

23. Pierog A, Martinez M, Mencin A. Outpatient endoscopic closure of persistent gastrocutaneous fistula with hemoclips in pediatric patients. Gastrointest Endosc. 2014;80(6):1190-3.

24. Farach SM, Danielson PD, McClenathan DT, Wilsey MJ, Chandler NM. Endoscopic closure of persistent gastrocutaneous fistula in children. Pediatr Surg Int. 2015;31:277-81.

25. Rodrígues-Pinto E, Pereira P, Vilas-Boas F, Macedo G. Over the scope clip closure of gastrocutaneous fistula. Rev Esp Enferm Dig. 2016; 108(9):578.

26. Jaramillo J, Hyun J, Abranjo C, Koppolu R, Chao S, Hartman G, et al. Salvage of a failed open gastrocutaneous fistula repair with an endoscopic over the scope clip. J Ped Surg Case Reports.2016;8:40-1.

27. Singhal S, Changela K, Culliford A, Duddempudi S, Krishnaiah M, Anand S. Endoscopic closure of persistent gastrocutaneous endoscopic gastrostomy (PEG) tube placement, using the over the scope clip system. Ther Adv Gastroenterol. 2015;8(4):182-8. 